Revista de Investigación de Física 23(3), (2020)

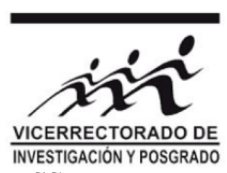

eISSN:1728-2977

\title{
Determinación del coeficiente de descarga de una compuerta plana deslizante de fuerte carga hidráulica
}

\author{
Bernardino Salvador Rojas ${ }^{1 *}$ \\ ${ }^{1}$ Universidad Nacional Mayor de San Marcos, Departamento Académico de Ingeniería Mecánica de Fluidos
}

Recibido 05 julio 2020 - Aceptado 14 diciembre 2020

\begin{abstract}
Resumen
Se reporta los resultados de un estudio de campo y gabinete para determinar el coeficiente de descarga $\left(C_{d}\right)$ de una "compuerta desgravadora" de sistema de almacenamiento y captación de agua de un proyecto hidroenergético. En la mayoría de los proyectos hidroenergéticos, el agua se capta desde una represa construida en el cauce de un río. Aguas abajo de la represa las condiciones de vida en el río se alteran. Para remediar esta situación, principalmente en épocas de estiaje, se libera agua desde la represa de manera controlada (caudal ecológico) operando, usualmente, la compuerta desgravadora. En ese contexto, el problema de los operadores del embalse está relacionado con la abertura de la compuerta que garantice el caudal ecológico para un determinado nivel de agua en el embalse. Para abordar esta problemática, se midieron el caudal aguas abajo de la compuerta para 3 aberturas relativas de ésta $(5 \%, 10 \%$ y $15 \%)$ y diferentes niveles de agua en el embalse. Con estos datos se hallaron valores de $C_{d}$. Los resultados muestran que $C_{d}$ tiende a aumentar para aberturas menores de $15 \%$. Esta tendencia podría estar influenciada por la geometría del labio inferior de la compuerta.
\end{abstract}

Palabras clave: Compuerta plana deslizante, compuerta desgravadora, coeficiente de descarga.

\section{Determination of the discharge coefficient of a heavy hydraulic head slide gate}

\begin{abstract}
The results of a field and office study to determine the discharge coefficient $\left(C_{d}\right)$ of a "sluice gate" of a water storage and intake system of a hydropower project are reported. In most hydropower projects, water is withdrawn from a dam built on a riverbed. Downstream of the dam the living conditions in the river are altered. To remedy this situation, mainly in times of low water, water is released from the dam in a controlled manner (ecological flow), usually by operating the sluice gate. In this context, the problem of the reservoir operators is related to the opening of the sluice gate that guarantees the ecological flow for a given level of the water in the reservoir. To solve this problem, the discharge downstream of the gate was measured for 3 gate relative openings $(5 \%, 10 \%$ and $15 \%)$ and different water levels in the reservoir. With these data $C_{d}$ values were found. The results show that $C_{d}$ tends to increase for openings smaller than $15 \%$. This tendency could be influenced by the geometry of the lower lip of the gate.
\end{abstract}

Keywords: Slide gate, sluice gate, discharge coefficient.

*bsalvadorr@unmsm.edu.pe 


\section{Introducción}

En obras de almacenamiento y derivación de agua, las compuertas se emplean como elementos de control. Por ejemplo, por medio de compuertas se mantiene el nivel de agua en el embalse; el caudal de derivación se controla por medio de compuertas; los materiales que decantan frente a las ventanas de captación se limpian operando "compuertas desgravadoras" (llamadas también "compuertas de limpia" o "compuertas de fondo").

Las compuertas desgravadoras, en su mayoría son compuertas planas deslizantes (slide gates) o también del tipo Broome (tractor gates). Y por estar ubicadas en la parte más baja de la presa soportan presiones altas. Por ello, se suele clasificar por la carga de presión $(H)$ que soportan: de baja carga $(H<15 m)$, de carga media $(15 m<H<30 m)$ y de alta carga $(H>30 m)$.

La compuerta desgravadora que motivó este trabajo forma parte de las obras de derivación de una Central Hidráulica $(\mathrm{CH})$. La presa se ubica a una altitud promedio de 1,300 msnm y forma un embalse en la cuenca media de un río de ceja de selva. En un represamiento en el cauce de un río como el presente caso, el control de las descargas de agua hacia aguas abajo de la represa es crucial, sobre todo en épocas de estiaje, ya que constituye el caudal ecológico establecido por Reglamentos. En este proyecto, la descarga del caudal ecológico se efectúa a través de la compuerta desgravadora.

En el contexto descrito, el problema de los operadores del embalse de la $\mathrm{CH}$ es determinar la abertura de la compuerta para un determinado nivel de agua en el embalse, que varía influenciado por diversos factores entre ellos el caudal de generación de la $\mathrm{CH}$, que también varía según el despacho de energía. Con el objetivo de solucionar esta problemática, primeramente se determinó el coeficiente de descarga $\left(C_{d}\right)$ de la compuerta desgravadora. Luego, con el $C_{d}$ hallado, se elaboró una tabla de caudales en función del nivel de la superficie de agua en el embalse y la abertura relativa de la compuerta. Esta tabla facilita la labor de los operadores del embalse al descargar el caudal ecológico.

\section{Marco Teórico}

\section{Esquema de ubicación de la compuerta}

La figura 1 es un corte longitudinal del túnel, de sección rectangular de1.8 m alto por $1.4 \mathrm{~m}$ de ancho, a través del cual se descarga el caudal ecológico. En el corte se muestra la ubicación de un par de compuertas que tienen el mismo ancho del conducto. Un esquema de la primera compuerta desgravadora, y la línea de gradiente hidráulica del sistema formado por el embalse, el conducto y la compuerta desgravadora sería como la Figura 2.

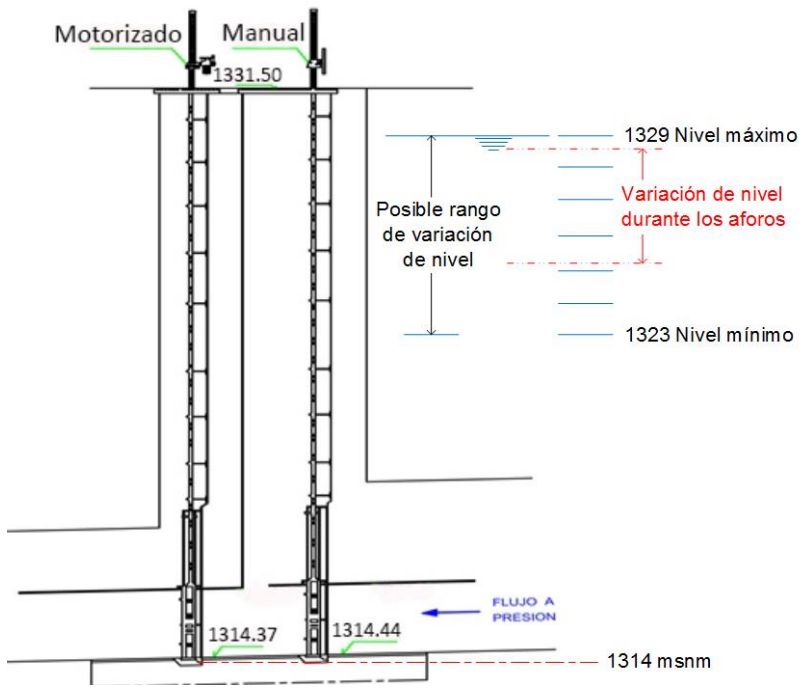

Figura 1: Ubicación de la compuerta desgravadora.

\section{Ecuación de la compuerta desgravadora}

Uno de los problemas de diseño más frecuente es la provisión de una capacidad de descarga adecuada en presas aliviaderos (libres o con compuerta), barrages, salidas, alcantarillas, etc. Las ecuaciones de descarga se basan invariablemente en las ecuaciones de continuidad y energía, o de momentum (Novak et al., 2010).

Cuando una compuerta plana funciona con cargas reducidas, el caudal descargado se puede calcular aplicando la teoría de orificios de grandes dimensiones (Acevedo et al., 1998). Sin embargo, en el presente caso la compuerta está ubicada dentro de un conducto a presión, a una cierta distancia aguas abajo de la entrada y sometida a una fuerte carga hidráulica. Por consiguiente, la fórmula de descarga se deriva a partir de las ecuaciones de continuidad y de energía.

En efecto, para el sistema (embalse, conducto y compuerta desgravadora) esquematizado en la Figura 2, la ecuación 1D de energía entre los puntos 1 y 2 , despreciando la pérdida de carga entre estos puntos, resulta

$H_{1}=y_{2}+\frac{V_{2}^{2}}{2 g}$

donde, $H_{1}$ (carga total en el punto 1) se iguala con la suma del tirante y la carga de velocidad en el punto 2 . 


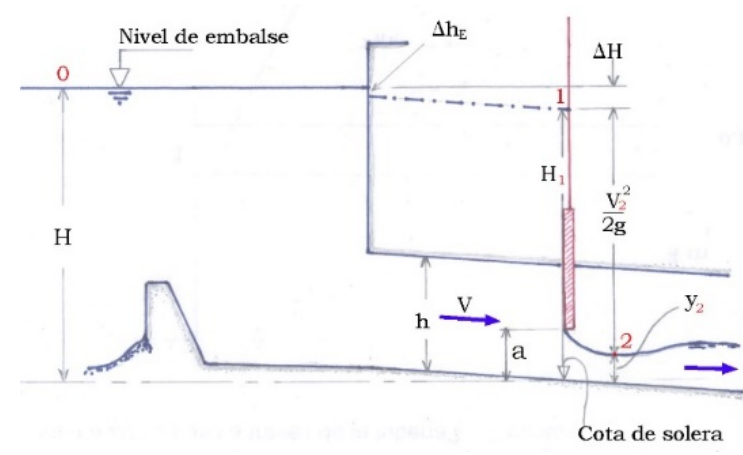

Figura 2: Esquema del sistema analizado.

Pero, la carga total en el punto 1 se puede expresar por

$H_{1}=H-\Delta H$

siendo $H$ la carga en el punto 0 (al despreciar la carga de velocidad); y $\Delta H$, la pérdida de carga total entre los puntos 0 y 1. En (2) la mayor dificultad está en la determinación de $\Delta H$. Sin embargo, para el esquema de la Figura 2 esta pérdida se puede expresar como

$\Delta H=\Delta h_{E}+h_{f}$

donde,

$\Delta h_{E}=K_{e} \frac{V^{2}}{2 g}$

Representa a las pérdidas locales de carga, principalmente en la entrada del conducto; y $h_{f}$ representa a la pérdida de carga por fricción, que puede calcularse con la siguiente fórmula empírica de Darcy:

$h_{f}=f \frac{L}{4 R} \frac{V^{2}}{2 g}$

En las fórmulas (4) y (5), $V$ es la velocidad media del flujo en el conducto; $K_{e}$ el coeficiente de pérdida local de carga, principalmente, por entrada; $L$ y $R$ son la longitud y el radio hidráulico del conducto, respectivamente.

Al reemplazar (4) y (5) en (3), y luego en (1), se obtiene:

$y_{2}+\frac{V_{2}^{2}}{2 g}=H-\left[K_{e}+f \frac{L}{4 R}\right] \frac{V^{2}}{2 g}$

Por otro lado, por continuidad:

$V A=V_{2} A_{2}$

donde $A=h B$ es el área del flujo en el conducto rectangular de alto $h$, y $A_{2}=y_{2} B$ es el área del flujo en la vena contracta (punto 2); siendo $B$ el ancho del conducto rectangular y de la compuerta. Entonces, de (7)

$V=\frac{y_{2}}{h} V_{2}$

Reemplazando (8) en (6); luego despejando $V_{2}$ :

$$
V_{2}=\sqrt{\frac{2 g\left(H-y_{2}\right)}{1+\left[K_{e}+f \frac{L}{4 R}\right]\left(\frac{y_{2}}{h}\right)^{2}}}
$$

Alternativamente, (9) se puede expresar en términos de las siguientes variables:

Abertura relativa de la compuerta: $\beta=\frac{a}{h}, 0 \leq \beta \leq 1$; siendo ala abertura de la compuerta y $h$ su altura.

Coeficiente de contracción del flujo a la salida de la compuerta: $C_{c}=\frac{A_{2}}{A_{o}}=\frac{y_{2}}{a}$, siendo $A_{o}=a B$ el área del orificio de la compuerta. De estas definiciones se obtiene:

$\frac{y_{2}}{h}=\beta C_{c}$

Entonces, al reemplazar (10) en (9) resulta

$$
V_{2}=\sqrt{\frac{2 g\left(H-\beta C_{c} h\right)}{1+\left[K_{e}+f \frac{L}{4 R}\right]\left(\beta C_{c}\right)^{2}}}
$$

Pero, la velocidad del flujo dada por (11) es teórica, ya que al plantear la ecuación (1) se despreció la pérdida de carga entre los puntos 1 y 2 . Sin embargo, con (11) se puede estimar la velocidad real $V_{2}^{*}$ como:

$V_{2}^{*}=C_{v} V_{2}$

donde $C_{v}$ es el coeficiente de velocidad. Así, el caudal descargado por la compuerta es: $Q=V_{2}^{*} A_{2}=C_{v} V_{2} y_{2} B$. $\mathrm{Y}$, teniendo en cuenta (10) y (11), resulta:

$$
Q=\beta C_{c} C_{v} h B \sqrt{\frac{2 g\left(H-\beta C_{c} h\right)}{1+\left[K_{e}+f \frac{L}{4 R}\right]\left(\beta C_{c}\right)^{2}}}
$$

\section{Coeficiente de descarga}

Debido a la dificultad de hallar los coeficientes $K_{e}$ y $f$ para el conducto, la ecuación (14) se puede reordenar como:

$Q=\frac{C_{c} C_{v}}{\sqrt{1+\left[K_{e}+f \frac{L}{4 R}\right]\left(\beta C_{c}\right)^{2}}} \beta h B \sqrt{2 g\left(H-\beta C_{c} h\right)}$

que también puede expresarse como

$Q=C_{d} \beta h B \sqrt{2 g\left(H-\beta C_{c} h\right)}$

donde,

$C_{d}=\frac{C_{c} C_{v}}{\sqrt{1+\left[K_{e}+f \frac{L}{4 R}\right]\left(\beta C_{c}\right)^{2}}}$

Basado en (17), se podría plantear la siguiente relación funcional para $C_{d}$ :

$C_{d}=F\left(C_{v}, C_{c}, K_{e}, f, \beta\right.$, forma $)$

y el método de análisis dimensional permitirá encontrar las variables adimensionales y la relación funcional de éstas. 


\section{Metodología}

\section{Trabajo de campo: medición de caudal}

Para medir el caudal se acondicionó un tramo del río aguas abajo de la presa. La sección hidrométrica fue dividida en 9 franjas de $0.6 \mathrm{~m}$ de ancho (franjas 2 al 10), y 2 franjas de $0.5 \mathrm{~m}$ de ancho (franjas 1 y 11 , en los extremos derecho e izquierdo, respectivamente), tal como puede observarse en el esquema de la figura 3 . La velocidad media de la franja se consideró a la velocidad medida en el eje vertical central de la franja, a 0.6 del tirante (medido desde la superficie libre del agua),

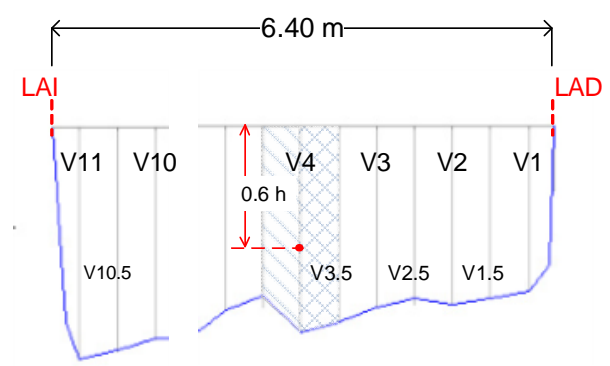

Figura 3: Esquema de la sección hidrométrica con detalles de la franja 4.

Se realizaron un total de 25 mediciones de caudal para tres aberturas relativas de la compuerta desgravadora $(5 \%, 10 \%$ y $15 \%)$. Las velocidades se midieron con correntómetro OTTC31 y OTTC2. Los caudales medidos se muestran en la tabla 1

\section{Trabajo de gabinete: Gráficas $Q$ vs. $\left(H-\beta C_{c} h\right)^{1 / 2}$}

En este trabajo, aun cuando las mediciones de caudal se realizaron para un rango de variación de $\left(H-\beta C_{c} h\right)$ corto, el coeficiente $C_{d}$ se calculó a partir de los caudales medidos (tabla 1). Para tal efecto, la ecuación (16) se expresó en la siguiente forma:

$Q=m\left(H-\beta C_{c} h\right)^{1 / 2}$

donde,

$$
m=C_{d} \beta h B \sqrt{2 g}
$$

Según la ecuación (19), los puntos de la gráfica $Q$ vs. $\left(H-\beta C_{c} h\right)^{1 / 2}$ deben emplazarse a lo largo de una recta con pendiente $m$. Por lo tanto, con el valor de $m$ obtenida a partir de la gráfica, $C_{d}$ se puede calcular desde (20), es decir,

$$
C_{d}=\frac{m}{\beta h B \sqrt{2 g}}
$$

Con los datos de la tabla 1 , se elaboraron gráficas $Q_{\text {medido }}$ vs. $\left(H-\beta C_{c} h\right)^{1 / 2}$ para cada valor de $\beta$. Para los cálculos, la carga total $H$ se determinó a partir de la siguiente relación:

$H=$ Nivel de embalse - Cota de solera de la compuerta
Sin embargo, porque las mediciones de caudal se realizaron sólo para un rango de variación de nivel del embalse corto (ver figura 1), se consideró necesario introducir un dato correspondiente al nivel aún más bajo. Este dato se calculó con la siguiente fórmula experimental de caudal para compuertas planas (Sotelo, 1997, p.215):

$Q=C_{d} B a\left(2 g h_{1}\right)^{1 / 2}$

Con $C_{d}=0.6$ y $\frac{h_{1}}{a} \geq 9$, siendo $h_{1}$ la altura delnivel de agua frente a la compuerta. Se tomó $H_{1}^{*}=h_{1}=9$ acomo el valor mínimo de $H_{1}$ para el cual se aplica la fórmula (23) con $C_{d}=0.6$ Las gráficas $Q_{\text {medido }}$ vs. $\left(H-\beta C_{c} h\right)^{1 / 2}$, muestran las figuras 4 y5. Es importante precisar que, para todos los cálculos se empleó $C_{c}=0.62$, valor recomendado para fines prácticos para cualquier relación $\frac{h_{1}}{a}$ inclusive para descarga sumergida (Sotelo, 1997).

Tabla 1.Caudales medidos para tres aberturasrelativas de la compuerta desgravadora.

\begin{tabular}{cccc}
\multicolumn{2}{c}{$\beta=0.05$} & \multicolumn{2}{c}{$\beta=0.10$} \\
\hline $\begin{array}{c}\text { N. de embalse } \\
(\mathrm{msnm})\end{array}$ & $\begin{array}{c}Q_{\text {medido }} \\
\left(\mathrm{m}^{3} / \mathrm{s}\right)\end{array}$ & $\begin{array}{c}\text { N. de embalse } \\
(\mathrm{msnm})\end{array}$ & $\begin{array}{c}Q_{\text {medido }} \\
\left(\mathrm{m}^{3} / \mathrm{s}\right)\end{array}$ \\
\hline 1325.01 & 1.430 & 1325.01 & 2.471 \\
1325.51 & 1.480 & 1325.51 & 2.619 \\
1325.81 & 1.465 & 1325.80 & 2.571 \\
1326.60 & 1.581 & 1326.61 & 2.708 \\
1326.85 & 1.533 & 1326.84 & 2.872 \\
1327.09 & 1.560 & 1327.10 & 2.602 \\
1327.15 & 1.574 & 1327.15 & 2.596 \\
1327.18 & 1.519 & 1327.19 & 2.718 \\
1327.26 & 1.410 & 1327.24 & 2.601 \\
1328.04 & 1.334 & 1328.10 & 2.600 \\
1328.72 & 1.279 & 1328.68 & 2.651 \\
\hline
\end{tabular}

\begin{tabular}{cc}
\multicolumn{2}{c}{$\beta=0.15$} \\
\hline $\begin{array}{c}\text { N. de embalse } \\
(\mathrm{msnm})\end{array}$ & $\begin{array}{c}Q_{\text {medido }} \\
\left(\mathrm{m}^{3} / \mathrm{s}\right)\end{array}$ \\
\hline 1325.03 & 3.507 \\
1325.51 & 3.603 \\
1326.59 & 3.711 \\
\hline
\end{tabular}

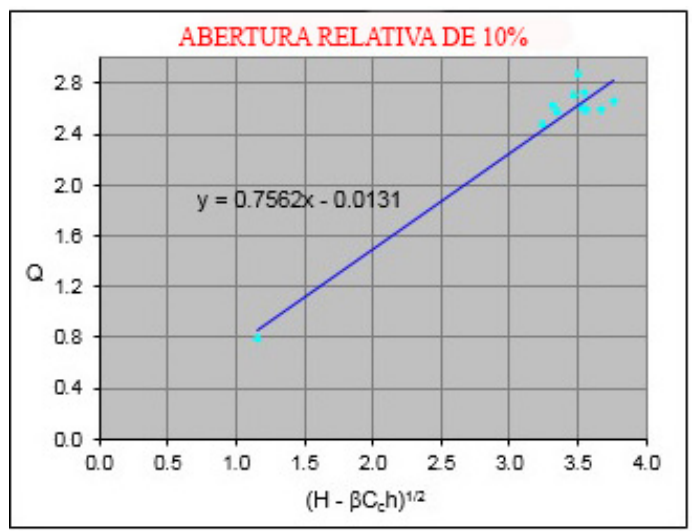

Figura 4. Abertura relativa al $10 \%$. 


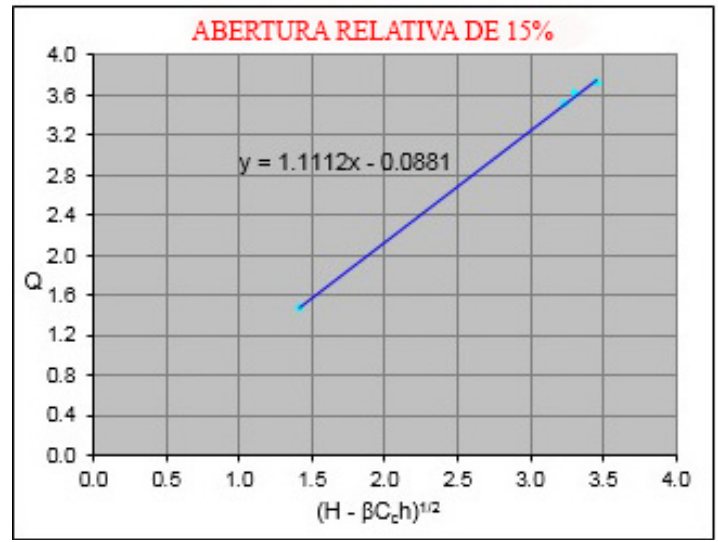

Figura 5. Abertura relativa al 15\%.

\section{Resultados}

\section{Coeficiente de descarga $C_{d}$}

Con los valores de la pendiente de la recta de ajuste (ver Figuras 4 y 5, por ejemplo), mediante la ecuación (21) se hallaron los coeficientes de descarga, para tres abertura relativas de la compuerta desgravadora (ver Tabla 2).

Tabla 2. Valores de la pendiente $m$ y $C_{d}$, para tres aberturas relativas de la compuerta desgravadora

\begin{tabular}{ccc}
\hline$\beta$ & $m$ & $C_{d}$ \\
\hline 0.05 & 0.414 & 0.74 \\
0.10 & 0.756 & 0.68 \\
0.15 & 1.111 & 0.66 \\
\hline
\end{tabular}

Tal como se observa en la Tabla 2, el coeficiente de descarga de la compuerta desgravadora aumenta cuando disminuye su abertura relativa. Para compuertas planas, la geometría de su labio inferior es un factor que contribuye a la variación del $C_{d}$ de este modo (ver por ejemplo, lámina $N^{\circ}$ A.8 deHydraulic Design Criteria, Sheet 320-1 -Corps of Engineers U.S. Army). En el caso de la compuerta desgravadora, el perfil de su labio inferior, originalmente plano, para la fecha de los aforos habría estado provisto de un sello en forma de "nota musical". En consecuencia, la tendencia de los valores de $C_{d}$ de la tabla 2 podría estar influenciada por la forma del sello empleado.

\section{Tablas nivel de embalse vs. caudal}

Con los valores hallados de $C_{d}$ (Tabla 2), se generaron tablas de Nivel de embalse (msnm) vs. $Q\left(\mathrm{~m}^{3} / \mathrm{s}\right)$. Para estos cálculos fue necesario expresar la carga total $H$ en términos de nivel de embalse. Para tal efecto, por la relación (22) y la cota de solera de la compuerta en 1,314.44 msnm, de (16) resultó la siguiente fórmula del caudal:

$Q=C_{d} \beta h B \sqrt{2 g\left[\text { Nivel de embalse }-\left(1,314.44+\beta C_{c} h\right)\right]}$ donde, $Q$ en $\mathrm{m}^{3} / \mathrm{s}, h=1.8 \mathrm{~m}, B=1.4 \mathrm{~m}$ y el Nivel de embalse expresado en msnm. Los caudales generados con la ecuación (24), para tres aberturas relativas de la compuerta desgravadora, muestra la Figura 6 . Tal como se observan en esta gráfica los caudales generados pasan entre los caudales medidos.

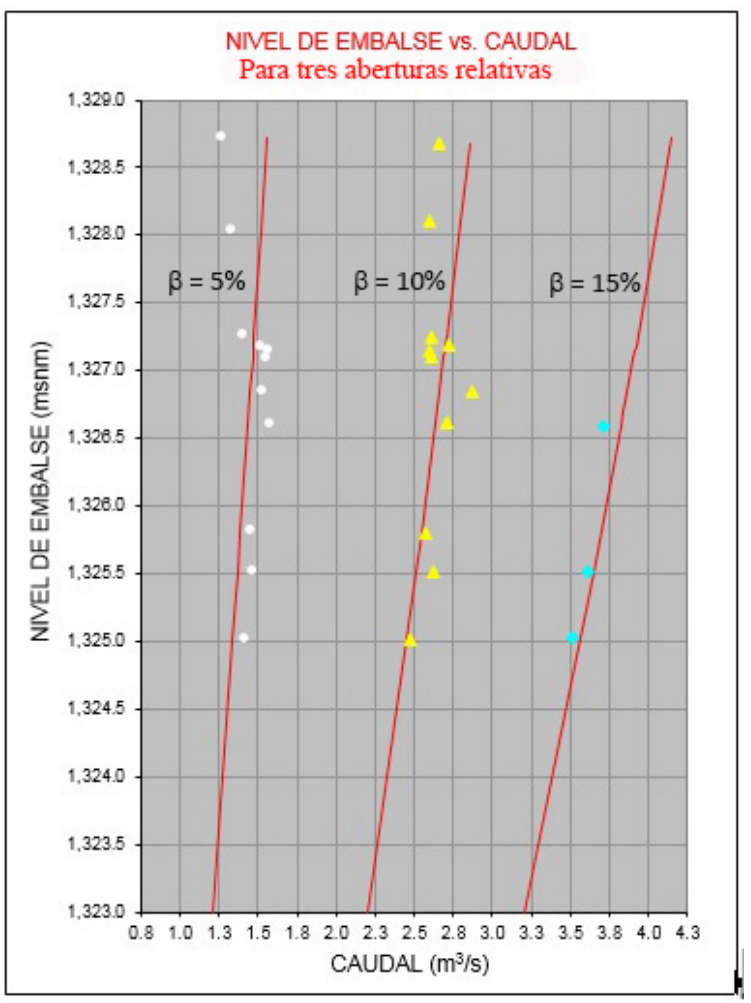

Figura 6: Caudales medidos (puntos blancos, amarillos y celestes) y caudales generados (líneas rojas continuas).

Tabla de caudales para los operadores del embalse

Considerando que los valores de $C_{d}$ de la tabla 2 son muy optimistas, se adoptó valores más conservadores de este coeficiente, los mismos que se presenta en la tabla 3.

Tabla 3. Valores de $C_{d}$ adoptados para la compuerta desgravadora.

\begin{tabular}{cc}
\hline$\beta$ & $C_{d}$ \\
\hline 0.05 & 0.70 \\
0.10 & 0.67 \\
0.15 & 0.65 \\
$>0.15$ & 0.65 \\
\hline
\end{tabular}

Finalmente, con la fórmula (24) y los valores de $C_{d}$ de la tabla 3, se elaboró una tabla Nivel de embalse (msnm) vs. $Q\left(\mathrm{~m}^{3} / \mathrm{s}\right)$ con abertura relativa $\beta$ de la compuerta desgravadora que van desde $5 \%$ hasta $100 \%$, con intervalos de $5 \%$. 


\section{Conclusiones}

Durante los trabajos de campo se realizaron un total de 25 aforos en cauce natural empleando correntómetro OTTC31 y OTTC2, para tres abertura relativas de la compuerta desgravadora $(\beta=0.05,0.10 y 0.15)$. Los aforos se realizaron sin interferir en lo absoluto a las condiciones de operación de la $\mathrm{CH}$.

Con los valores de caudal medidos, se han calculado los coeficientes de descarga $C_{d}$ de la compuerta desgravadora. Los resultados muestran valores altos tanto como 0.74 para $\beta=5 \%$, disminuyendo a 0.68 y 0.66 para coeficiente de abertura de $10 \%$ y $15 \%$, respectivamente. Esta tendencia de variación de $C_{d}$ se observa, en general, para compuertas planas deslizantes sometidas a alta carga hidráulica; donde los valores altos para aberturas pequeñas están influenciados por la forma del labio inferior de la compuerta.

Por las condiciones particulares bajo las cuales se realizaron los trabajos de campo (aforos en pleno funcionamiento de la $\mathrm{CH}$, variación de la superficie libre en el embalse sólo en un rango pequeño), para elaborar la tabla de caudales descargados por la compuerta desgravadora se ha adoptado valores de $C_{d}$ más conservadores (ver tabla 3 ).

Con valores conservadores de $C_{d}$, se ha elaborado tabla Nivel de embalse (msnm) vs. $Q\left(\mathrm{~m}^{3} / \mathrm{s}\right)$, con aberturas relativas de la compuerta desgravadora que van desde $5 \%$ hasta $100 \%$, con intervalos de $5 \%$, para que puedan usar los operadores de embalse de la $\mathrm{CH}$.

\section{Referencias}

Acevedo, J. (1998). Manual de Hidráulica. São Paulo: Edgard Blücher.

Department of The Army, Corps of Enginners. Hydraulic Design Criteria, Sheet 320-1. U. S. Army Enginner Waterways Experiment Station, Vicksburg, Mississipi. s/e.

Department of the Army, Corps of Enginners. Engineering Manual (1985). Washington, D.C. s/e.
Lomax, W. y Saul, A. (1979). Laboratory Works in Hydraulics. London: Granada Publishing Limited.

Novak, P. (2010). Hydraulic Modelling - an Introduction. Principles, methods and applications. London: Spon Press.

Sotelo, G. (1997). Hidráulica General, Volumen 1 Fundamentos. México: Limusa S.A. 